\title{
DESTRUCTION OF METALLIZATION SYSTEMS ON SILICON FROM OVERHEATING TO MELT MIGRATION
}

\author{
Arkady A. Skvortsov ${ }^{1}$, Sergey M. Zuev², Pavel A. Skvortsov ${ }^{3}$ \\ 1,2,3 Mechanics of Materials Department \\ Moscow Polytechnic University \\ 107023, Bolshaya Semenovskaya Street 38, \\ Moscow, RUSSIAN FEDERATION
}

\begin{abstract}
The article introduces the analysis of thermal degradation mechanisms related to the heating and melting of metallization system, as well as the contact fusion in transient conditions, and carries out an investigation of the thermal damage of metallization systems under the influence of current pulses. The method of pulse impact on the structure of the metal-semiconductor. The results showed that both thickness and thermal conductivity of the underlayer strongly influence the dynamics of heating of the multilayer system. The results obtained during the experiments show that the various heat-conducting properties of the $\mathrm{SiO} 2$ and Si3N4 films are clearly reflected in the connection oscillograms. As a result of defects, a local reduction of the film cross-section leads to an increase in current density, temperature, and consequently, appearance of a local molten area. Electron microscopy confirmed the presence of contact melting at the interface Al-Si. Phase analysis conducted in the border region has shown that the amount of Si impurities in the eutectic makes about $63 \%$. The occurrence of the interface temperature gradients leads to stress, which contributes to the formation of structural defects near the source of thermal shock. The resulting temperature gradient causes the thermoelastic critical condition that could lead to a violation of the adhesion of the metal film, the formation of structural defects and failure of the test compound. The experimental results of thermal degradation of metallization systems, as well as the calculation of physical indicators are described.
\end{abstract}

$\begin{array}{lr}\text { Received: } & \text { April 4, } 2017 \\ \text { Revised: } & \text { April 4, } 2017 \\ \text { Published: } & \text { May 8, } 2017 \\ \text { } \text { Correspondence } & \text { author }\end{array}$

(c) 2017 Academic Publications, Ltd. url: www.acadpubl.eu 
AMS Subject Classification: $14 \mathrm{H} 02,14 \mathrm{~A} 02,51 \mathrm{P} 02$

Key Words: thermal destruction, metallization systems, melt migration, semiconductors, silicon

\section{Introduction}

At the moment, the share volume of $\mathrm{R} \& \mathrm{D}$ at the microelectronics market (estimated as $\$ 348$ billion in 2015) comes to $8-10 \%$, or about $\$ 35$ billion in cash. The high investment cost provokes the interest of researchers to this sector of economics, resulting in a significant technological breakthrough in this direction.

The main trends of modern microelectronics are reducing the topological size of models, shifting into low gear power consumption and improving the quality of products. All mentioned above is impossible without a detailed analysis of the structures composing the modern microelectronic devices. The thermal degradation analysis of metallization systems and semiconductor structures and contacts becomes the most important component of such investigations [1]. This issue has been studied by many scientists.

Hyung Giun Kim and T.A. Nguyen in their research are contemplating a simple test structure $[2,3]$ which allows capturing just an integral characteristic of the entire track [4]. The initial stages of degradation processes as well as spreading of melted zones (speed and direction) are almost impossible to be recorded on structures of this type.

Nowadays, no analysis of degradation mechanisms (in terms of their separation) under nonstationary actions in metal-semiconductor systems is represented in scientific studies. For example, melting and directed contact fusion mechanisms in these structures develop almost simultaneously, but the literature does not describe experimental procedures which allow their separation.

Moreover, the degradation processes may be accompanied by the formation and subsequent proliferation of fused areas both on the surface and in the bulk of the semiconductor crystal. This aspect, in relation to the micro- and nanoelectronics structures, isn't treated thoroughly. The development of such methods is particularly relevant from the point of view of their application to new conductive materials as well.

At present, many attempts have been being made to create new types of metallization systems, including nanoelectronics structures: $\mathrm{Cu}$, titanium silicide TiSi2 [5]. 


\section{Materials and Methods}

Analyzing the contact pairs with regard to new composition and geometry, one needs to pay attention primarily to the plot $\lambda\left(\Delta t_{1}\right)$ on a pilot $U$-shaped curve of reliability [6], which represents the dependence of $\lambda$ bounce from the time of operation of metallization $t$.

$\lambda\left(\Delta t_{1}\right)$ plot is a test site, where metallization systems of semiconductor structures (burn-in testing, thermal cycling) are tested. In cyclograms of such tests a profile of operating external exposure factors on the studied structures is repeated in an accelerated form.

In the segment $I-\lambda\left(\Delta t_{1}\right)$ the reliability of the metallization, a degree of defect-free manufacturing and yield rate of products are revealed. This segment is to be minimized, as it requires a significant expenditure of resources, to get to the area of stable operation of products $I I-\lambda\left(\Delta t_{2}\right)$. Regarding to the section $I I I-\lambda\left(\Delta t_{3}\right)$ of the curve, it goes beyond the effective life of the product as a whole and is related to the termination of operation of products thereby it is not analyzed in detail [7].

Apart from the selection of materials for metallization systems, the operating conditions of structures are of great importance as well. Operation of metallization systems and contacts in conditions of high electrical and thermal load contributes to their accelerated degradation.

It should be noted that despite an active search of new materials for metallization systems aluminium keeps being the most widespread material. Therefore, the aim of this article is to study the thermal degradation of metallization systems including mechanisms occurring in the metal-semiconductor structures under pulse action starting from the aluminum metallization heating up to the formation of molten zones and their migration.

For registration of temperature changes in the near-surface layers of silicon a test structure in a form of metallization track $(A 1)$ was applied to the semiconductor surface by the technique [1]. Originally rectangular-shaped pulses with the electric power $\left(P>3.0 \div 5.0 .109 \mathrm{~A} / \mathrm{m}^{2}\right)$, which doesn't destroy the structure were passed through the plot. We are talking about the common thermal heating of a metallization system [8]. Besides rectangular pulses, other forms of current pulses were used (pulses with a ramp up leading edge, sinusoidal).

An aluminium metallization with proportions $100 \times 200 \mu m$, coated on a silicon substrate, was used as a sample. The thickness of aluminum metallization is $1 \div 3 \mu \mathrm{m}$, the thickness of the semiconductor substrate $350 \div 400 \mu \mathrm{m}$. Besides aluminum films, polycrystalline copper films with a similar dimension type sprayed onto the silicon plates were studied. 
For consideration of thermal modes of metallization systems, inclusion oscillograms $U(t)$ as described in [1] were analyzed. Its meaning is as follows: during the pulse advancing $I(t)$ (supplied with special sondes) the voltage drop $U(t)$ from a plot of the test is removed by the corresponding probes. The shape of the current pulse and waveforms was fixed by a digital storage oscilloscope. In this case temperature dynamics of the metallization track $T(t)$ was calculated by the change in the voltage $\operatorname{drop} U(t)$ :

$$
\alpha \cdot\left(T(t)-T_{0}\right)=\frac{U(t)}{I(t) \cdot R_{0}}-1
$$

where $R_{0}$ - resistance of the metallization track at $T_{0}=290 \mathrm{~K}$, measured by a voltmeter-ammeter; $\alpha$-temperature dependent drag coefficient. Equation (1) allows us to experimentally analyze the dynamics of the temperature dependent operation of the metal-semiconductor interface $\Delta(t)=T(t)-T_{0}$.

In the study of metal-semiconductor systems with different sublayers the equation previously obtained in [1] was used:

$$
\Delta T(t)=T_{1}(t)-T_{0}=\frac{I^{2} \bar{R}_{1}}{S}\left[\frac{h_{2}}{\overline{\lambda_{2}}}+\frac{1}{\bar{c}_{3} \bar{d}_{3}} \sqrt{\frac{t}{\bar{a}}}\right]
$$

where the subscript " 1 " corresponds to the Al-film; "2" - to a dielectric thin film with a thickness $h_{2}, m$; "3" - to a semiconductor matrix. Where $I-$ current, $A ; \bar{R}_{1}$ - metallization path resistance, $\Omega ; S$ - heat transfer area, $m^{2}$; $\lambda_{2}$ - silicon thermal conductivity coefficient, $W /(m \cdot K) ; t$ - time, $s ; c_{3}$ - heat capacity, $J /(k g \cdot K)$; $d_{3}$ - density, $\mathrm{kg} / \mathrm{m}^{3}$, a-thermal diffusivity, $\mathrm{m}^{2} / \mathrm{s}$. The bar over the corresponding symbol means the average integral (temperature dependent) value.

\section{Results and Discussion}

The results showed that both thickness and thermal conductivity of the underlayer strongly influence the dynamics of heating of the multilayer system. The results obtained during the experiments show that the various heat-conducting properties of the $\mathrm{SiO} 2$ and $\mathrm{Si} 3 \mathrm{~N} 4$ films are clearly reflected in the connection oscillograms. Dynamics of the metallization track heating is shown in Figure 1. 


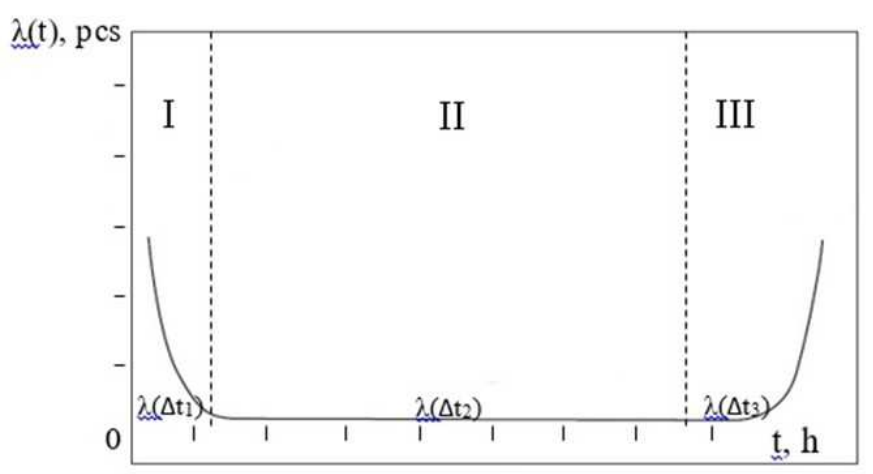

Figure 1: $U$-shaped curve of the reliability of metallization systems of modern semiconductor devices and structures.

The results showed that the dynamics of heating of the multilayer system is strongly influenced by both thickness and thermal conductivity of the underlayer. It is easy to see that a variety of heat-conducting properties of the $\mathrm{SiO} 2$ and Si3N4 films is clearly reflected in the connection oscillograms (Figure 2).

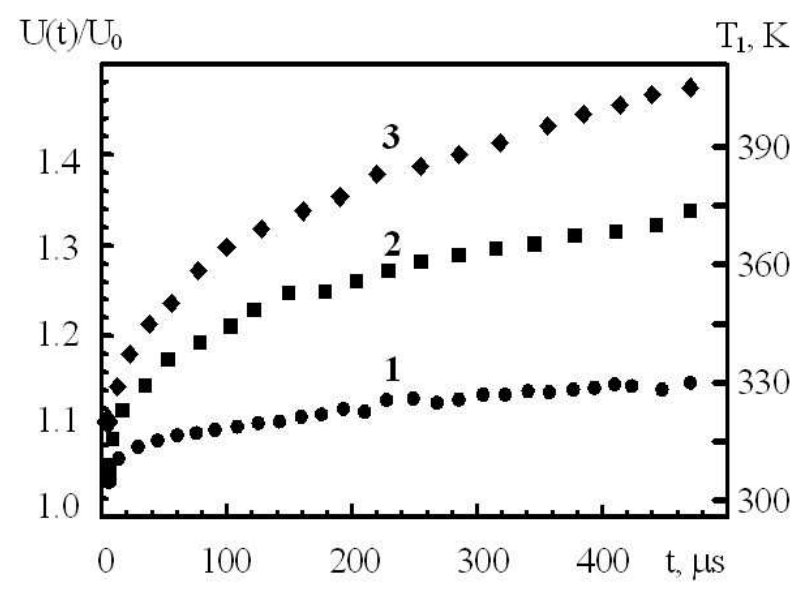

Figure 2: Waveforms of connection of the system $S i$-insulator- $A l$ while passing the single-pulse current with an amplitude $j=3 \cdot 10^{10} \mathrm{~A} / \mathrm{m}^{2}$ and a duration of $440 \mu s, h_{1}=5 \mu s: 1-h_{2}=0 ; 2-S i_{3} N_{4} ; h_{2}=0.5 \mu m ; 3-S_{i O}$;

$$
h_{2}=0.5 \mu \mathrm{m} \text {. }
$$

Previously, it was revealed [1] that degradation processes when exposed to rectangular current pulses in metallization systems based on aluminium are connected with formation of fused areas (reaching the melting point of the metal 
film) and contact fusion at the interface $\mathrm{Al}-\mathrm{Si}$. The last one begins to appear when reaching the system's eutectic point (Figure 3 ).

$O B$ plot (similar O'B 'and O"B") in Figure 3 is characterized by a monotonic increase of $U(t)$ with a relatively small time $\mathrm{t}$ after the pulse which is related to the heating of the structure (of a thin metal film) and is determined by the regime of the heat removal to a semiconductor plate meanwhile power per time unit allocated in the aluminum does not manage to be completely discharged into the silicon substrate, whereby the structure is heated.

In sections $\mathrm{AB}, \mathrm{A}^{\prime} \mathrm{B}$ ', $\mathrm{A}$ " $\mathrm{B}$ " of these structures the starting of the phase formation was recorded, shown in the oscillograms as a sharp deviation from the monotonic growth (potential jump), which is related to the processes of melting aluminum (melting point of $A l=661^{\circ} \mathrm{C}$ ), i.e. the formation of the molten zone and its spreading along the metallization track.
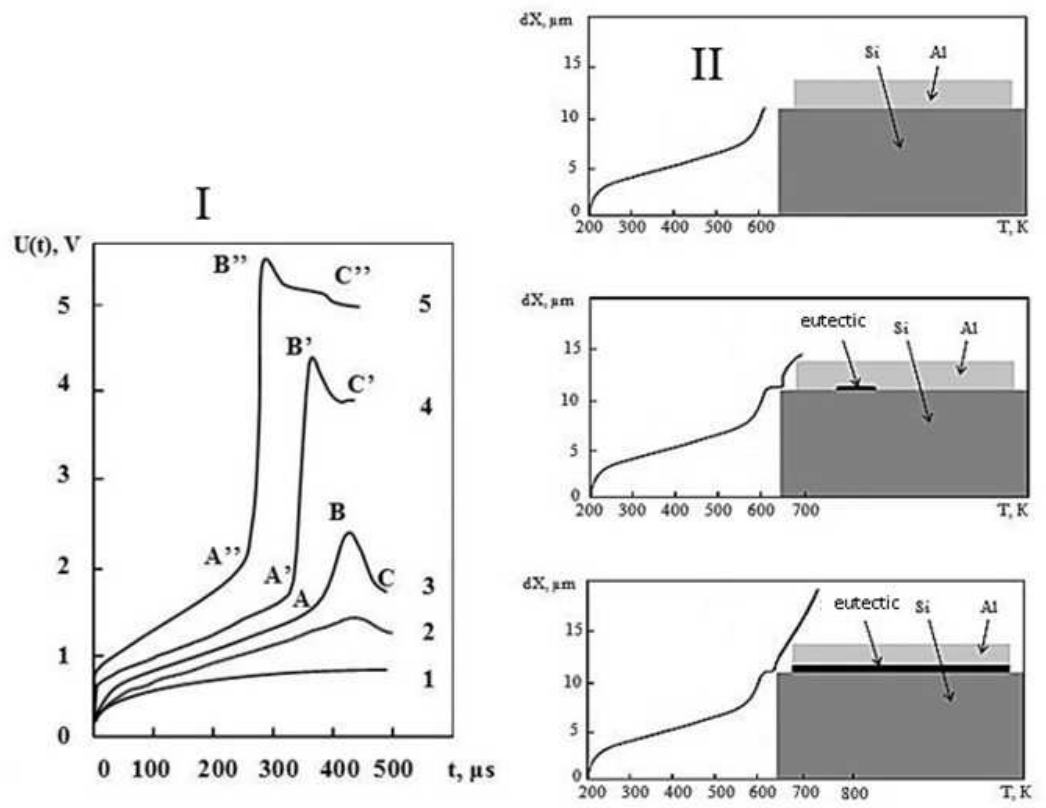

Figure 3: $I$ - A view of connection waveforms $U(t)$ during the passage of a single current pulse through an aluminum metallization track lying on silicon.

The pulse duration $\tau_{i}=500 \mathrm{~ms}$, the amplitude $j\left(\mathrm{~A} / \mathrm{m}^{2}\right): 1-4.5 \cdot 10^{10} ; 2-$ $5.0 \cdot 10^{10}$ (contact fusion, without melting $\mathrm{Al}$ ); $3-5.2 \cdot 10^{10}$ (contact melting, partially melting $A l$ ); $4-5.6 \cdot 10^{10}$ and $5-5.8 \cdot 10^{10}$ (complete melting of $A l$ ).

$I I$ - the view of the waveform type according to the applied load.

Plot BC, B'S 'B"S' 'is characterized by the contact fusion process at the 
aluminum-silicon interface: potential growth and its subsequent "reset" (eutectic point of the system $A l-S i$ is $577^{\circ} \mathrm{C}$ ) and extension of the conductive layer. These processes are developed at current densities (called critical) $j \sim 6 \cdot 10^{10} \mathrm{~A} / \mathrm{m}^{2}(\tau>150 \mathrm{~ms}$, the resistance of the structure at room temperature $\left.R_{0}=0.8 \Omega\right)$.

The photos of destruction during a single rectangular current pulse propagation through the aluminum metallization track lying on silicon are shown in Figure 4.

In most cases, the test structure geometry violation occurs in places of poor adhesion of the film to the semiconductor. Indeed, experimental tests confirm that the adhesive strength of the samples depends on the dimensions (thickness, width) of the structure, as well as the direction and speed of application of an external current load. During the adhesion, weak in comparison with the cohesion, an adhesive separation occurs. In a case of relatively weak cohesion a cohesive rupture of the adhesive substance is possible.
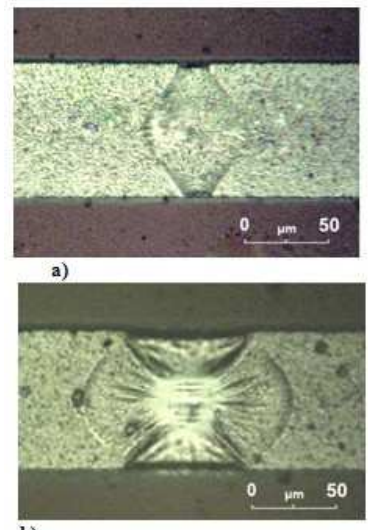

b)

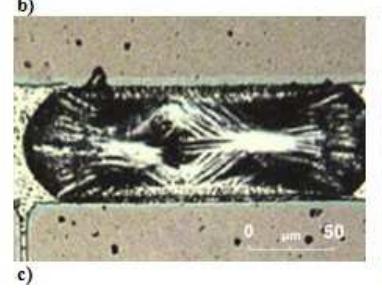

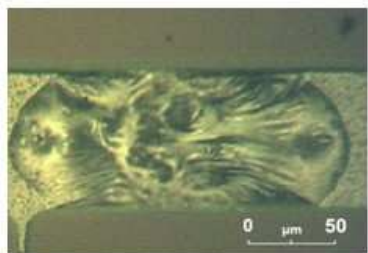

d)
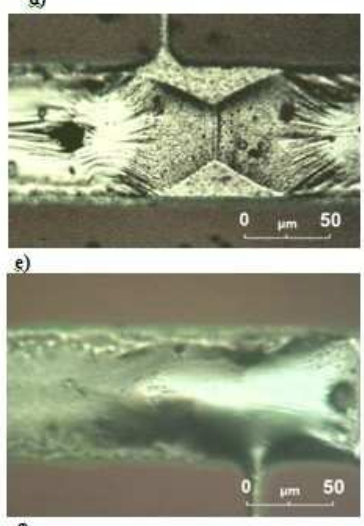

Figure 4:Photographs of the test structure fragments after rectangular current pulse propagation with duration of $500 \mu s$ and an amplitude of: $a-j=5.0 \cdot 10^{10} \mathrm{~A} / \mathrm{m}^{2}$ (the initial stage of the melted zone formation corresponds to curve 2, Figure 2 ); $b-j=5.1 \cdot 10^{10} \mathrm{~A} / \mathrm{m}^{2}$ (development of the melted zone); c, $d-j=5.2 \cdot 10^{10} \mathrm{~A} / \mathrm{m}^{2}$ (the formation of a melted zone and the beginning of its transfer corresponds to curve 3, Figure 3); 
$e j=5.6 \cdot 10^{10} \mathrm{~A} / \mathrm{m}^{2}$ (dynamics of the melted area, including processes of

"meeting" of adjacent molten portions, corresponds to curve 3, Figure 3); $f j=5.8 \cdot 10^{10} \mathrm{~A} / \mathrm{m}^{2}$ (melting of the entire structure corresponds to the curve 4 in Figure 3). The width of the track is $75 \mu \mathrm{m}$.

On the scale this violation of the examined film geometry is shown as a waveform $U(t)$ peak drift to the right. This drift of the waveform maximum point with potential unloading from various parts of the test track is shown in Figure 5. This distribution characterizes a time shift in the spreading of the liquid film.

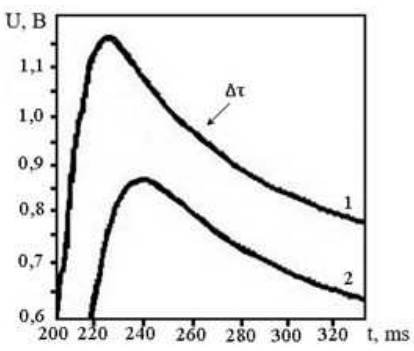

Figure 5: Waveforms $U(t)$, taken from two sections of test structure of $A l-S i$ system during the single rectangular current pulse propagation with an amplitude of $5.8 \cdot 10^{10} \mathrm{~A} / \mathrm{m}^{2}$ and a duration of $480 \mu \mathrm{s}$. The signal was taken from sites with a length of $660 \mu \mathrm{m}$, located at $1.5 \mathrm{~mm}$ (curve 1) and $1.8 \mathrm{~mm}$

(curve 2) of the negative electrode; $\Delta \tau=22 \mu \mathrm{s}$.

Let us thoroughly consider the mechanism of fused areas formation and the direction of their propagation in metallization systems.

We associate the formation of fused areas with electrotransport and thermodiffusion processes in defective parts of the metal tracks. For samples that were studied in this work multistage tunnel-recombination processes involving surface states [2] can be considered as a main mechanism of current transport.

As an example, let's investigate the effect of current transport to the metallization path when passing a current pulse with a duration of $\tau=600 \mu \mathrm{s}$ and $j=3.0 \cdot 10^{10} \mathrm{~A} / \mathrm{m}^{2}$. Aluminium diffusion coefficient is $D=1.7 \cdot 10^{-5} \mathrm{~cm}^{-2}$. The shift of the substance during the pulse propagation can be calculated by the formula:

$$
x=2 \cdot \sqrt{D \cdot \tau}
$$

As a result we find that after the current impact the displacement $x$ is about $2.0 \cdot 10^{-5} \mathrm{~cm}$, which is a negligibly small quantity. 
Regarding the thermal diffusion, it should be noted that the thermal impact on the silicon surface causes the formation of strong temperature gradients that lead to high mechanical stresses. The recrystallization process at the substratemetallization boundary the diffusion of $S i$ to the aluminum layer occurred, which also leads to the stress formation. Whereby linear defects in the region of maximum gradient in the $\{111\}$ plane of the plates are formed.

During the thermodiffusion of a two-layer system under the assumption that the external forces are equal to zero, and the pressure is the same at all points of a test sample:

$$
D_{12} \nabla c_{1}+D^{\prime}{ }_{12} c_{1}\left(1-c_{1}\right) \nabla T=0
$$

where $c_{1}$ is the concentration of $A l, m^{-3} ; D_{12}-$ usual diffusion coefficient, $\mathrm{m}^{2} / \mathrm{s} ; D_{12}^{\prime}$ - thermal diffusion coefficient, $\mathrm{m}^{2} / \mathrm{s}$.

The ratio of the thermal diffusion coefficient to usual diffusion coefficient is described by the equation:

$$
s_{T}=\frac{D_{12}^{\prime}}{D_{12}}=-\frac{\nabla c_{1}}{\nabla T} \frac{1}{c_{1}\left(1-c_{1}\right)}
$$

where $s_{T}$ - Soret coefficient [6].

In addition to poor adhesion of the film to the substrate, the thermoelectric properties are affected by the volume defects of the structure. One of the common causes of such defects in metal-dielectric substrate systems are dust and foreign debris coming from the environment in the manufacturing process (moving in the manufacturing zone, lithography, etc.). It is also possible that Si particles breakaway and get to the surface when handling plates during manufacturing operations. It should be noted that oxide packaging faults, resulting in increased current leakage and failure of circuits, isolated hillocks in epitaxial layers, pinholes in insulating films may also be regarded as defects [10].

As a result of defects, a local reduction of the film cross-section leads to an increase in current density, temperature, and consequently, appearance of a local molten area.

Formation of such a section when $j \sim 4.0 \cdot 10^{10} \mathrm{~A} / \mathrm{m}^{2}$, usually occurs at the early stages $(\sim 50 \mu s)$ of the pulse propagation. With a current density increasing to $j \sim 8.0 \cdot 10^{10} \mathrm{~A} / \mathrm{m}^{2}$ and current pulse durations $\tau_{i}>100 \mu \mathrm{s}$ such sites can spread along the metallization tracks. The direction and the average speed of the melted area displacement $V(t)$ during the propagation of the current pulse were estimated by the dynamics of oscillograms (Figure 3). Knowing the distance between sites, which were filmed with oscillograph, and time, one can estimate the value of $V$. For the applied pulses this value was $10-50 \mathrm{~m} / \mathrm{s}$. 
It is obvious that the molten zone undergoes various changes, both geometric (increase of the size of the molten zone) and thermochemical (diffusion), in the process of a current pulse propagation.

Distribution of the molten zone related to the heat emission on its boundary was experimentally fixed (Figure 6).

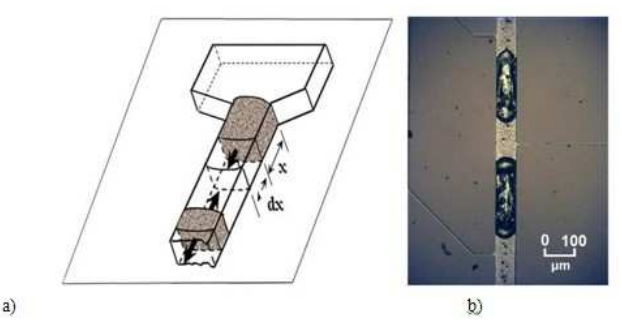

Figure 6: Scheme of spreading of melted zones in the aluminum metal track (a), an experimental model (b). On the photo - areas of a structure meltback after the propagation of the single current pulse with an amplitude of $7.0 \cdot 10^{10} \mathrm{~A} / \mathrm{m}^{2}$ and a duration of $400 \mu \mathrm{s}$, a pulse energy of $64 \mathrm{~mJ}$.

The boundary of the solid $(s)$ and the liquid $(l) \mathrm{Al}$ is an area of coexistence of phases having a finite thickness $l_{x}$. Unbalanced heat emission in this area can be calculated by the formula:

$$
\left[I(\tau)^{2}\left(\rho_{o l}-\rho_{o s}\right) \frac{l_{x}}{S}\right] d \tau=H \cdot S \cdot d x
$$

where $I(\tau)=k \cdot \tau ; \rho_{o l}=2.4 \cdot 10^{-7}, \Omega \cdot m ; \rho_{o s}=0.98 \cdot 10^{-7}, \Omega \cdot m$ - the resistivity of the liquid and solid aluminium, $b=75 \mu \mathrm{m}, h=5 \mu \mathrm{m}$ - width and thickness of the tracks, respectively, $S=b \cdot h$-cross-sectional area of conductor.

If we consider that $W_{s l}$ (at the aluminium melting point) is used for melting certain volume of metal $S \cdot d x$, we have:

$$
W_{s l} \cdot d \tau=\cdot H \cdot S \cdot d x
$$

where $H=10.2 \cdot 10^{8} \mathrm{~J} / \mathrm{m}^{3}$ - specific heat of aluminium melting, $d x$ - length of the melted part of the track, which was formed during the current pulse propagation with $W_{s l}$ output during the melting time $d \tau$, and from (6) and (7) it follows that:

$$
\left[I(\tau)^{2} \cdot\left(\rho_{o l}-\rho_{o s}\right) \frac{l_{x}}{S}\right] \cdot d \tau=H \cdot S \cdot d x
$$

The last form can express the length of the fused area:

$$
d x=\frac{I^{2}(\tau)\left(\rho_{o l}-\rho_{o s}\right) \sqrt{a_{1} \tau}}{S^{2} H} d \tau
$$




$$
\begin{gathered}
x=\frac{\left(\rho_{o l}-\rho_{o s}\right) \sqrt{a_{1}}}{S^{2} H} \int_{0}^{\tau} I^{2}(\tau) \sqrt{\tau} d \tau \\
x=\frac{\left(\rho_{o l}-\rho_{o s}\right) \sqrt{a_{1}}}{S^{2} H} \int_{0}^{\tau} k^{2} \tau^{2} \sqrt{\tau} d \tau \\
x=\frac{\left(\rho_{o l}-\rho_{o s}\right) \sqrt{a_{1}}}{S^{2} H} k^{2} \int_{0}^{\tau} \tau^{\frac{5}{2}} d \tau .
\end{gathered}
$$

Let $l_{x}$ commensurable with the thickness of the layer within which heat is distributed during the exposure time: $l_{x}=2 \sqrt{a_{1} \tau}=(1-4) \cdot 10^{-4} \mathrm{~m}$ (the ratio is usually used for impulse effects of different kinds: laser, electric, etc.), where $a_{1}=1.6 \cdot 10^{-5} \mathrm{~m}^{2} / \mathrm{s}$ - thermal diffusivity of aluminium, $\tau$ - fragment $[0 \ldots x]$ of metallization track fusion time (counted from the moment A - or A', A" on the oscillograms of Figure 3).

Then, integration of the equation (12) allows us to obtain the dependence of the length of the fused metallization track $x_{l}$ from the time $\tau$ for the surge current with linearly rising edge:

$$
x(\tau)=\frac{\left(\rho_{o l}-\rho_{o s}\right) \sqrt{a_{1}}}{S^{2} H} k^{2} \frac{2}{7} \tau^{\frac{7}{2}} .
$$

In the case of a rectangular current pulse:

$$
x(\tau)=\frac{\left(\rho_{o l}-\rho_{o s}\right) \sqrt{a_{1}} I_{0}^{2}}{S^{2} H} \tau^{\frac{3}{2}}
$$

A verification of equations $(13,14)$ was made on Al-SiO2-Si and Al-Si structures. For that single current pulses with an amplitude $j>5.0 \cdot 10^{10} \mathrm{~A} / \mathrm{m}^{2}$ and duration $\tau_{i}>200 \mathrm{~ms}$ were passed through these structures. Upon reaching the "critical" current densities on the surface of the aluminum film the appearance of molten zones was observed. The formation of such areas was haphazard so they appeared in various places of the test structure. However, regardless of the place of origin, interphase boundaries with $\tau_{i}>200 \mu s$ started moving. After turning off the pulse, the melted portions crystallized.

From equation (14) the dependence of the resistivity of molten aluminium on the contact boundaries with $\mathrm{Si}$ and $\mathrm{SiO} 2$ can be determined:

$$
\rho_{o l 2}=\rho_{o s}+\eta\left(\rho_{o l 1}-\rho_{o s}\right)
$$


where $\eta$ - the tangent of the inclination angle in the coordinates of dependence of the length of the fused area on the melting time (Figure 6).

Experimental results for systems of Al-SiO2-Si and Al-Si with a rectangular current pulse (Formula 14) are shown in Figure 7.

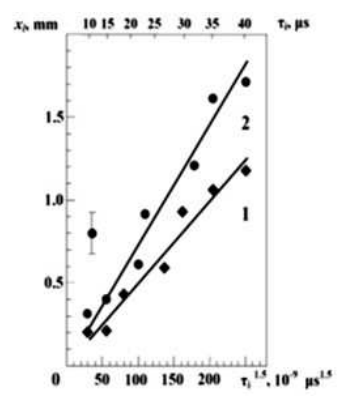

Figure 7: Dependence of the length of the fused area on melting time $\tau$ for systems: 1 - Al-Si, 2 - Al-SiO2-Si. The amplitude of the current pulse $j=6.0 \cdot 10^{10} \mathrm{~A} / \mathrm{m}^{2}$, and $\tau_{i}$ duration varied from 300 to $500 \mu \mathrm{s}$.

It is easy to see that, given the numerical values of the parameters used, the value of $x_{l}$ is consistent with the experiment for both Al-Si binary system and the multi-layer structure Al-SiO2-Si, that is the evidence in favor of the mechanisms described above.

However, attention is drawn to the difference in the dynamics of structures' reflow (straight lines tilt angles in Figure 6). In our opinion, this is determined by the different conditions of heat removal during the current pulse propagation, as well as the possibility of current bridging by near-surface layers of the semiconductor in the system Al-Si. Indeed, in the area of considered temperatures $\left(\sim 660^{\circ} \mathrm{C}\right)$ in a binary system, a true current density is lower the calculated one in comparison with a system with a dielectric underlayer because of current bridging bynear-surface layers of the semiconductor. Therefore, at the equal electric power of acting pulse, the size of the molten aluminium film portion in the system Al-SiO2-Si is larger than that in the binary system. Especially clearly this distinction is observed when melting time $\tau>35 \mathrm{~s}$.

Speaking about the possible impact of contact melting processes, it should be emphasized that in the context of the experiment carried out at Al-Si structures, directed fusion mechanisms prevail over contact melting in the initial stage of heating of structures (for example, see Figure 3, curves 3, 4, 5) waveform portion $\mathrm{OA}$ - heating of the metal film, $\mathrm{AB}$ - fusion, and further - contact melting (BC section).

The mechanisms of contact and directed fusion depend on the duration and amplitude of the pulse passed through the sample. For example, if you impose 
a pulse of long duration $\tau$ but a small current amplitude $I$ through the plating, then there is the prevalence of contact reflow. On the other hand, if the pulse duration $\tau$ is relatively small compared with the value $I$, then the structure will be dominated by the directional reflow mechanism.

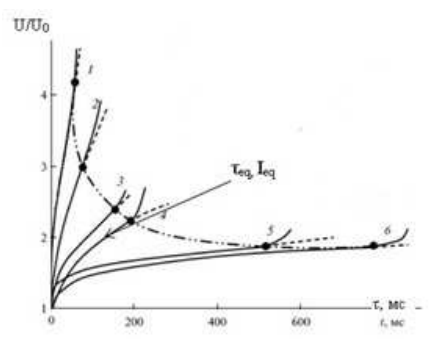

Figure 8: View of inclusion waveforms accompanying degradation processes in the system Al-Si, during the propagation through the structure of a single current pulse with an amplitude: $1-j=8.8 \cdot 10^{10} / \mathrm{m}^{2} ; 2-$

$$
\begin{gathered}
j=8.6 \cdot 10^{10} \mathrm{~A} / \mathrm{m}^{2} ; 3-j=8.2 \cdot 10^{10} \mathrm{~A} / \mathrm{m}^{2} ; 4-j=6.7 \cdot 10^{10} / \mathrm{m}^{2} ; 5- \\
j=6.6 \cdot 10^{10} / \mathrm{m}^{2} ; 6-j=6.1 \cdot 10^{10} \mathrm{~A} / \mathrm{m}^{2}
\end{gathered}
$$

This was observed experimentally. It is clear that for certain values $\tau_{e q}$ and $I_{e q}$ there will be a balance of contact and directed plating reflow mechanisms. Experience has shown that for Al-Si structure $\tau_{e q}$ and $I_{e q}$ data were obtained (Figure 8) by propagating rectangular pulses with $j=66.1 \times 10^{10} \div 8.8 \times$ $10^{10} / m^{2}$ [1]. Curve 4 in Figure 8 is very different from the curve 5 by the angle of inclination, which means that at the given pulse parameters with $j=$ $6.7 \times 10^{10} \mathrm{~A} / \mathrm{m}^{2}$ degradation mechanisms (directed fusion), which differ from the degradation mechanisms implemented at pulse transmission with $j=6.6 \times$ $10^{10} / \mathrm{m}^{2}$ (contact melting), are prevailing in the structure.

In addition to the analysis of the process of fused in situ areas formation, it is important to have data on the state of the sample after crystallization of melted areas of the metal film.

Due to this, the electron microscopic studies were performed (Figure 9) on the two-beam scanning electron microscope Helios NanoLab 660, allowing measurements in high vacuum conditions $6 \times 10^{-4} \mathrm{~Pa}$ with an accelerating voltage of $500-30000 \mathrm{~V}$. During the examination the depth and the structure of the recrystallized layer were investigated. 

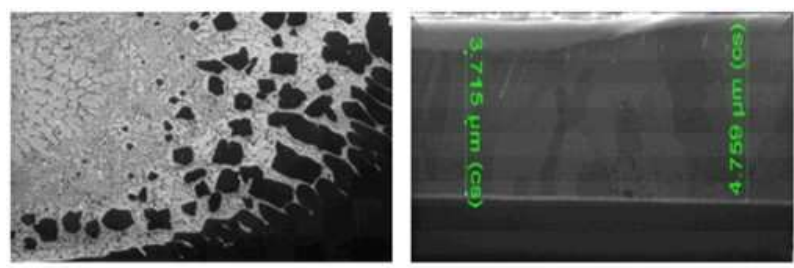

Figure 9: Photographs of the melted portions of test structures, obtained with a scanning electron microscope Helios NanoLab 660: left - the structure of crystallized eutectic $\mathrm{Al}-\mathrm{Si}$; right - the structure of the interface

$$
\mathrm{Al}-\mathrm{SiO}_{2}-\mathrm{Si} \text {. }
$$

Electron microscopy confirmed the presence of contact melting at the interface Al-Si.

Phase analysis conducted in the border region has shown that the amount of Si impurities in the eutectic makes about 63\% (Figure 10).

Worth noting that the occurrence of the interface temperature gradients leads to stress, which contributes to the formation of structural defects near the source of thermal shock, this fact requires further research. Through analysis of the thermoelastic state by the method proposed in [12], based on the calculation of thermoelastic stress in binary systems according to formulas (16), (17), the numerical calculation results were obtained for the study of structures (Figure $11)$ :

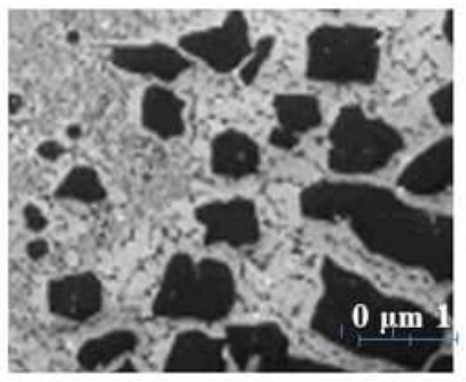

Figure 10: To the phase analysis in the border area of a crystallized eutectic Al-Si. 


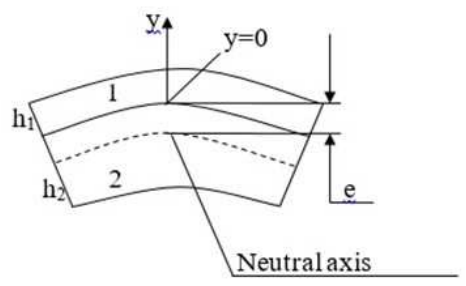

Figure 11: The calculation of thermal stress in binary systems.

Where: 1 - copper layer, 2 - ceramic layer

$$
\begin{aligned}
& \sigma_{1}(T)=\frac{\left(\alpha_{1}(T)-\alpha_{2}(T)\right) \Delta T}{\left(1 / E_{1} h_{1}+1 / E_{2} h_{2}\right)\left(1 / h_{1}+\left(h_{1}+h_{2}\right) E_{1} y /\left(2\left(E_{1} I_{1}+E_{2} I_{2}\right)\right)\right)} \\
& \sigma_{2}(T)=\frac{\left(\alpha_{1}(T)-\alpha_{2}(T)\right) \Delta T}{\left(1 / E_{1} h_{1}+1 / E_{2} h_{2}\right)\left(1 / h_{2}-\left(h_{1}+h_{2}\right) E_{2} y /\left(2\left(E_{1} I_{1}+E_{2} I_{2}\right)\right)\right)}
\end{aligned}
$$

where $\alpha_{i}$-temperature coefficient of linear expansion; $E_{i}$ - modulus of elasticity; $\Delta T_{i}$ - temperature difference; $I_{i}$ - moment of inertia of the detail crosssection, where $i=1,2$ (hereinafter, subscript 1 refers to the metal layer, respectively, and 2 - to the ceramic layer).

$$
\begin{aligned}
& I_{1}=\left(\left(h_{1}+e\right)^{3}-e^{3}\right) / 3, \\
& I_{2}=\left(\left(h_{2}-e\right)^{3}+e^{3}\right) / 3
\end{aligned}
$$

where $e$ is the distance from the interface surface to the neutral layer:

$$
e=\left(E_{2} h_{2}^{2}-E_{1} h_{1}^{2}\right) /\left(2\left(E_{1} h_{1}+E_{2} h_{2}\right)\right) .
$$

Where $y$ is the distance from the considered point to the neutral layer (positive direction down).

For Al-Si system using formulas (16), (17) the numerical value of thermoelastic stress were obtained. In this area of the temperature drop between the aluminium layer and the silicon $\Delta T=10-100 K$ voltages are linear (Figure 12).

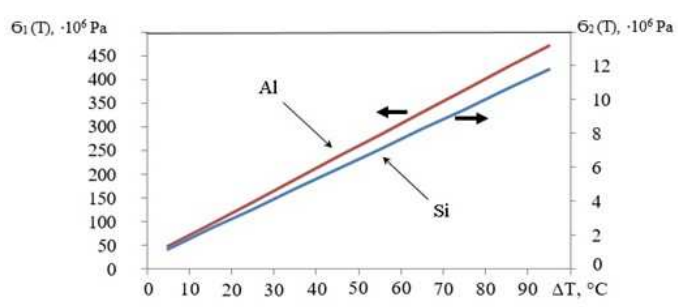

Figure 12: Calculation of thermoelastic stress in binary systems. Here: 1 calculation for the aluminium film, 2 - for the silicon substrate. 
Stresses in the aluminium film are significantly higher than the voltage in the aluminium layer, which suggests the possibility of further degradation capacity of the system, for example, when in conditions that differ from normal. Thus, if such a system is under a low temperature, Al-film gets warmed considerably faster than Si substrate at the pulse propagation. The resulting temperature gradient causes the thermoelastic critical condition that could lead to a violation of the adhesion of the metal film, the formation of structural defects and failure of the test compound, which requires a separate study.

\section{Acknowledgement}

This work was financially supported by the Ministry of Education and Science of the Russian Federation (projekt No 8.5171.2017).

\section{References}

[1] A. Skvortsov, S. Zuev, M. Koryachko, and V. Glinskiy. Thermal shock in metallization systems on silicon and the degradation of metallization systems and contacts, Microelectronics International, 33(2), (2016), 102-106.

[2] H.-G. Kim, D.-H. Cho, E.-K. Jeong, et al. Microstructure Evolution of Al-1\% Si Bonding Wire for Microelectronic Reliability. Electronic Materials Letters, 5(3), (2009), 99-103.

[3] T.A.Nguyen, D. Labrousse, P.-Y. Joubert, S. Lefebvre, and S. Bontemps. Study on aluminium reconstruction and bond wire lift-off effects on current density distribution in power semiconductor dies. PCIM Europe, Nuremberg, (2013).

[4] H.-G. Kim, T.W. Lee, S.M. Kim, S.Z. Han, K. Euh, W.Y. Kim, and S.H. Lim. Effects of $\mathrm{Ti}$ addition and heat treatments on mechanical and electrical properties of $\mathrm{Cu}-\mathrm{Ni}-\mathrm{Si}$ alloys. Metals and Materials International, 19(1), (2013), 61-65.

[5] A.A. Skvortsov, S.G.Kalenkov, and M.V. Koryachko. Phase transformations in metallization systems under conditions of nonstationary thermal action. Technical Physics Letters, 40, (2014), 787-790.

[6] A.A. Skvortsov, A.V. Karizin, L.V. Volkova, and M.V. Koryachko. Effect of a constant magnetic field on dislocation anharmonicity in silicon. Physics of the Solid State, 57, (2015), 914-918.

[7] A.A.Skvortsov, S.M. Zuev, and M.V. Koryachko. Metallization systems at pulse current influence. Phase transitions in the conditions of thermal shock. APEDE 2014 International Conference on Actual Problems of Electron Devices Engineering, 2, (2014), 333-340.

[8] A.A. Skvortsov, S.M. Zuev, and M.V. Koryachko. Electrothermal degradation of systems of metallization at non-stationary current influences. APEDE 2014 International Conference on Actual Problems of Electron Devices Engineering, 2, (2014), 340-343.

[9] V.I. Staroselsky. Physics of semiconductor devices in microelectronics. Moscow: URAIT Higher Education, (2009). 
[10] S.P.Timoshenko, B.M.Simonov, and V.N. Goreshko. Fundamentsls of reliability theory. Moscow: URAIT Hither Education, (2015).

[11] O.A. Kuznetsov, and A.I. Pogalov. Durability of soldered joints. Moscow: Mashinostroenie, (1987)

[12] V.V. Brus, M.I. Ilashchuk, Z.D. Kovalyuk, and P.D. Maryanchuk. Semicond. Sci. Technol., 27, (2012). 055008. 
